\title{
The Challenge of Doing What Is Right in Renal Transplantation: Balancing Equity and Utility
}

\author{
Aisling E. Courtney Alexander P. Maxwell \\ Regional Nephrology Unit, Belfast City Hospital, Belfast, UK
}

\section{Key Words}

Kidney transplantation - Ethics · Utility $\cdot$ Equity $\cdot$ Allocation

\begin{abstract}
Arguably the greatest challenge faced by the transplant community is the disparity between the number of persons waiting for a solid organ transplant and the finite supply of donor organs. For renal transplantation the gap between supply and demand has risen annually reflecting the increasing prevalence of end-stage renal disease versus the relatively static deceased donor organ pool. Maximising the benefit from this scarce resource raises difficult ethical issues. For most patients on dialysis therapy a successful transplant offers improved quality and quantity of life, but the absolute gain in survival provided by a donated organ varies greatly depending on recipient factors such as age and comorbid illnesses. The philosophies of equity (a fair opportunity for everyone in need to receive a transplant) and utility (optimal profit from each organ) are often competing. $\mathrm{Na}$ tional allocation schemes and local policies regarding assessment of potential recipients and acceptance of organs are designed to balance these ethical principles in a standardized and socially acceptable manner. The ongoing debate surrounding these issues and modifications to such policies reflect the evolving clinical picture of renal transplantation and the challenge in maintaining equipoise between renal transplant utility and equity.
\end{abstract}

Copyright $\odot 2008$ S. Karger AG, Basel

\section{KARGER}

Fax +4161306 1234 E-Mail karger@karger.ch www.karger.com

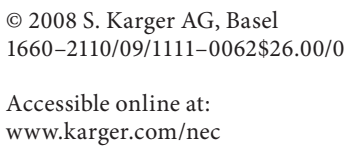

\section{Introduction}

Renal transplantation offers a survival advantage over dialysis therapy for the majority of persons with endstage renal disease (ESRD) [1], and is economically advantageous compared to other forms of renal replacement therapy (RRT). However, the disparity between the number of people waiting for a renal transplant and the finite supply of donor organs continues to increase.

Initially, limited by resources, there were stringent acceptance criteria to chronic dialysis therapy and to being listed for a renal transplant. Historically, our region was comparable to others in excluding those under 16 and over 50 years of age, or with multisystem disease including diabetes mellitus (DM). Gradually, these exacting criteria have been relaxed and there are now very few absolute contraindications to RRT. The rising prevalence of obesity, with associated diabetes mellitus and hypertension, coupled with the changing age demography of the population, is driving the increase in the prevalent dialysis patient population in the UK (www.renalreg.org).

Inevitably, the number of patients waiting for a kidney transplant is rising, with a $36 \%$ increase in UK waiting list numbers within the last decade. In addition, both the age and co-morbid illnesses of those on the waiting list are considerably greater than in previous decades (www. uktransplant.org.uk). In the UK, on 31 March 2008, 6,784 patients were waiting for a kidney transplant, with only

Aisling E. Courtney

Regional Nephrology Unit, Level 11-Belfast City Hospital

Lisburn Road

Belfast BT9 7AB (UK)

Tel. +44289032 9241, Fax +44 289026 3535, E-Mail aecourtney@doctors.org.uk 
1,437 deceased donor and 829 living donor kidney transplants performed in the previous year.

To address this shortage of kidneys for transplantation there has been a promotion of living donation, including unrelated donor/recipient pairs, increased use of extended criteria donors, and ongoing development of nonheart-beating donor programmes. Despite these measures currently only a third of the patients on the waiting list in the UK and one in five of those in the USA [2] are transplanted each year. The appropriate and just allocation of organs remains a relevant issue and the subject of considerable debate.

A variety of options are available when considering the rationing of a scarce resource. These include distribution on the basis of social worth or merit, according to the ability to pay (commoditisation), by queue (first come first served), by lottery (chance), and by maximum benefit (greatest good to the greatest number). This final method of allocation has been considered by the international transplant community to be the most appropriate; however, maximising the use of a precious resource such as donated kidneys raises difficult questions about the utility of renal transplantation versus equity of access. The utilitarian argument advocates that each kidney should be transplanted into the recipient in whom it will survive the longest [2]. The principle of equity or justice demands that each person who would benefit from a renal transplant should have comparable opportunity to receive one [3] (fig. 1).

A compromise between these two potentially conflicting ethical principles is essential in clinical practice, at an individual level as well as in national and international agreements. From the Hippocratic Oath (first do no harm) to the current professional ethical standards (render to each patient a full measure of service and devotion), a personal responsibility to do the best for each individual patient has been and is required of clinicians. The dilemma arises as the allocation of a kidney to the benefit one person inevitably does 'harm' to another who cannot therefore receive that transplant and continues to wait for an appropriate organ.

\section{Challenges to Utility}

In view of the scarcity of organs, the debate about how best to utilise this resource is important. The impressive survival rates of the first 100 renal transplants in Northern Ireland, UK (1968-1976), when all recipients were less than 50 years old and did not have any systemic disease

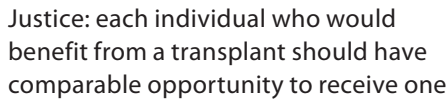

Justice: each individual who would

benefit from a transplant should have

comparable opportunity to receive one
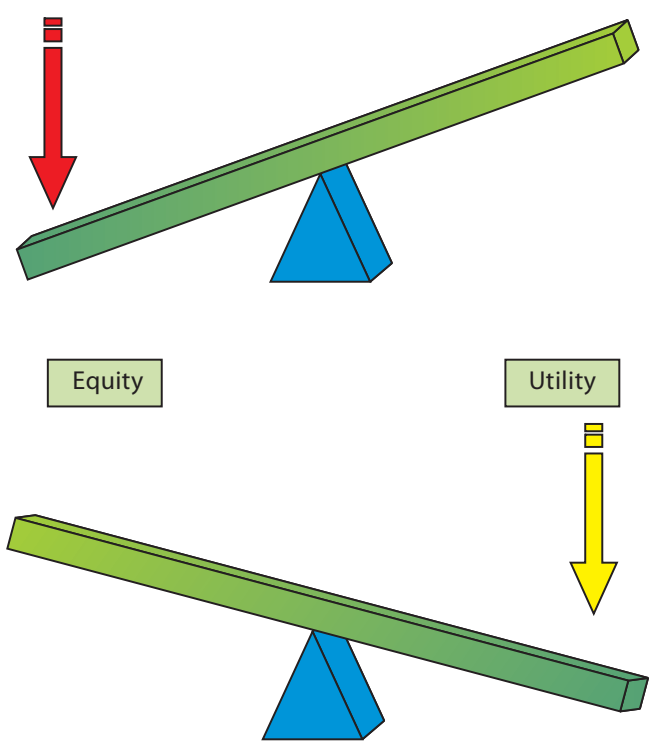

Each kidney should be transplanted into the recipient in whom it will survive the longest

Fig. 1. Ethical equipoise in transplantation.

is worth consideration [4]. The 5-year graft survival exceeded $82 \%$. The most recent average deceased donor graft survival rate amongst UK transplant centres is $81 \%$ (www.renalreg.org) despite improved knowledge of immunobiology and advances in immunosuppressive pharmacology. To adopt a strict utilitarian position and achieve the best outcome for each kidney, based on this data, those over 50 years old and those with DM would not be listed for a transplant. The lack of substantial improvement in long-term deceased donor kidney allograft survival over the past two decades reflects, at least in part, the changing demography of the persons now receiving renal transplants.

Age

In 2006, half of all new incident renal replacement therapy patients in the UK were over 60 years of age (www.renalreg.org). There is substantial support for transplantation in this age group with evidence that it is both safe and successful with life expectancy exceeding that on dialysis for appropriately selected patients. Argu- 
ably preclusion from renal transplantation on the basis of age alone is not fair and selection should be on the basis of biological fitness rather than chronological age [5]. However, undeniably life expectancy for older transplant recipients is shorter than that for younger patients, the 'gain of years' compared to remaining on dialysis is less than that for younger recipients, and whether death with a functioning graft is 'a success' is equivocal. However, with the progressive change in age demographics in developed countries, the perception that those over 60 years of age are 'elderly' will increasingly be challenged as would refusal to consider whether or not to undertake transplantation on the criterion of age alone.

\section{Diabetes Mellitus}

Reduced recipient survival in those with pre-existing DM has been consistently reported, particularly with type 2 diabetes. Death-censored graft survival is usually comparable with other groups and the poorer outcome is attributed to increased cardiovascular events. Nevertheless, the prognosis for patients with DM on dialysis therapy is poor, and even adjusting for co-morbid factors it remains a significant risk factor for death in the dialysis population (www.renalreg.org). The projected survival benefit from transplantation compared to continuing on dialysis therapy in patients with diabetic nephropathy exceeds that of other primary renal diseases with an estimated gain of 11 years [1].

\section{Obesity}

The impact of pre-transplant obesity on renal transplant outcomes is uncertain. Nevertheless, the largest registry data available for both short-term outcomes, such as delayed graft function and acute rejection [6], and overall survival [7] suggest that being obese at the time of transplantation is associated with an increased risk of early complications and a reduction in both recipient and death-censored graft survival. No studies have demonstrated whether a prospective reduction in weight prior to transplantation favourably alters risk, although many transplant centres have a local policy precluding transplantation if the potential recipient's body mass index exceeds a pre-specified limit. Such individuals have a significantly increased mortality risk if they remain on dialysis treatment rather than receive a transplant [8].

\section{Other Co-Morbidities}

Pre-existing cardiovascular disease is increasingly common in wait-listed patients concurrent with the rise in age; prevalence of DM, obesity, and associated hyper- tension; and time on dialysis prior to transplantation. This has a negative impact on recipient survival after transplantation [9]. Although debate persists concerning the optimal tool for assessing cardiovascular disease in potential recipients, such investigations have primarily been concerned with the fitness of the individual for the surgical procedure rather than the projected long-term survival after successful engraftment.

This is in contrast to the approach to potential recipients who have had previous malignancy, where life expectancy predictions are an important aspect of decision making. Such persons are generally precluded from being wait-listed until a reasonable disease-free period has passed after which the risk of recurrence is considered acceptable. The Israel Penn International Transplant Tumour Registry (www.ipittr.org) is the source of much of the data in this area.

\section{Challenges to Equity}

\section{Ancestry/Ethnicity}

Non-Caucasian patients waiting for a deceased donor kidney transplant are disenfranchised by an under-representation in the donor pool and an over-representation (compared to the general population) in the ESRD population, together with an allocation policy that emphasizes human leukocyte antigen (HLA) matching [10]. This results in longer waiting times before transplantation. The inequity produced by strict HLA matching criteria has been recognised in several countries resulting in modifications of national allocation schemes.

\section{Immunological Barriers}

Patients with high levels of panel reactive antibodies (PRA $>85 \%$ ) have a much smaller pool of potential donors than those that are not highly sensitized, and subsequently wait longer for an acceptable graft. Pre-transplant desensitization programmes designed to remove donor-specific HLA antibodies have been introduced to facilitate transplantation in such individuals. While undoubtedly increasing the transplant rate in this cohort it is a resource intensive process (similar protocols to allow $\mathrm{ABO}$ incompatible living donor transplants result in an increased cost of USD 38,000 compared to ABO-compatible procedures [11]), with substantial short-term risks and the literature on the long-term outcomes remains scanty. From a utilitarian perspective this is not the best use of limited resources (both financial and deceased donor organs). The case for justice may also question the prioritisation given 
to highly sensitized persons; if such individuals have already had one or two or more kidney transplants, is it fair that they get priority for another? Yet is it fair, that without special provision, such individuals would have little prospect of receiving a deceased donor organ?

\section{Geographical Location}

Geographical disparity in the access to transplantation within a country has been reported internationally. There is variability in gaining entry to the transplant waiting pool and the duration of time spent there [12]. The percentage of prevalent dialysis patients (aged less than 65 years) listed varies from 16 to $52 \%$ in UK renal units. It seems improbable that age and ethnic mix accounts for all of this variation; differences in assessment and acceptance onto the waiting list must exist despite attempts to standardize this process with national and international guidelines on recipient assessment (www. uktransplant.org.uk). Substantial disparities also exist in the median waiting time to deceased donor transplant for adults in the UK once they are listed; for the period of 2001-2004 the range was 399-1,905 days. Highlighting these discrepancies may potentially lead to investigation and minimisation of such inequalities.

\section{Ability to Pay}

For most transplant clinicians the fact that those who can afford to pay for a kidney are able to do so is abhorrent. However, within the international transplant community there are those that provide such a service. In the context of a global shortage of donated organs there is a growing industry of organ sales and transplant tourism with patient-tourists from rich countries traveling abroad to buy an organ from economically poorer people. The principle of equity is challenged by those whose socioeconomic status affords them the opportunity for a transplant that is denied to persons unable to buy an organ. Others would argue that the latter group would also benefit from such an arrangement as each live unrelated transplant that removes a person from the waiting list for a deceased donor organ will increase the chances of those remaining in the pool of receiving a transplant.

However, ethical consideration cannot be applied only to potential recipients but also to donors. A summit meeting concerning organ trafficking and transplant tourism in 2008 resulted in 'The Declaration of Istanbul' with the statement that 'organ trafficking and transplant tourism violate the principles of equity, justice and respect for human dignity and should be prohibited' (http://www. eurotransplant.nl/files/misc/declaration.pdf) [13].

The Challenge of Doing What Is Right in Renal Transplantation
This consensus is not the unanimous opinion amongst the transplant community, and the debate persists concerning both organ trafficking and also the compensated and regulated living unrelated donor transplant programme that was introduced in Iran in 1988. While this is the only country that has eliminated the waiting list for kidney transplantation, concern remains regarding the potential compromise of ethical principles in this system.

\section{Bias within Allocation Schemes}

The injustice that certain groups wait longer for a deceased donor kidney due to age, race, gender, geographical location, or socioeconomic status may be compounded by the suggestion that some of these cohorts (those older than 65 years and non-Caucasians) also receive suboptimal organs [14]. One report also suggested that obese patients are likely to wait longer for a transplant irrespective of all other factors implicated in allocation, and it was proposed that this bias may reflect the greater risk and smaller profit margins associated with transplanting such individuals [15].

\section{National Allocation Policies}

National allocation schemes are designed with the aim of minimizing inequalities in access, achieving medical utility, and maximizing efficiency in terms of logistics and cost in renal transplantation. Although there is now legal opportunity for altruistic non-directed living donation in the UK, the overwhelming number of living donors have at least an emotional if not a genetic link to the recipient and as such allocation is not an issue. Likewise, it is not viable to delay engraftment of non-heart-beating donor organs and these kidneys are used locally at the place of retrieval. However, repeated modification of national allocation schemes for deceased donor organs reflects the ongoing challenge of achieving equipoise between the utilitarian and egalitarian philosophies.

In both the UK and USA the allocation system is based on a points score for each recipient. In the USA the overwhelming factor determining the allocation of deceased donor organs is waiting time, and this is 'trumped' primarily only by zero HLA mismatched organs (less than $20 \%$ of kidneys) or by child recipients (approximately $10 \%$ of kidneys) [16]. Some are concerned that this system emphasizes justice to the detriment of utility as a kidney from a young donor can as readily be transplanted into an older recipient with limited post-transplant life expec-

Nephron Clin Pract 2009;111:c62-c68 
tancy as into a recipient of comparable age who needs a kidney with projected longevity.

In 2006, a new allocation system was implemented in the UK (www.uktransplant.org.uk). The previous system was designed to maximise the number of recipients that received a well-matched graft, based on the scientific literature demonstrating better graft survival for such transplants; however, those with an unusual tissue type waited for a disproportionately long time before being transplanted. Less emphasis is now placed on matching because of this adverse effect on equity and the realization that non-immune factors can have at least as great an impact on long-term graft outcomes. The new policy still prioritises complete HLA matching while at least theoretically making it easier for persons with less common tissue types to receive a kidney transplant, and has a points' scheme that is weighted in favour of well-matched kidneys for younger adults. In addition to the degree of HLA matching and recipient age, points are also accrued for each day spent on the waiting list, and are given to recipients that are close geographically to the retrieval centre for a particular organ, so the ischaemic time is minimized.

Future modifications are likely with the aim of further improving fairness, utility and efficiency. All with interest in renal transplantation need to be cognizant that no allocation system increases the number of donated organs, and as long as they remain a scare resource, the conflict between maximizing the benefit for an individual versus all of society will persist.

\section{The Patients' Perspective}

There is little information available about the patients' perspective on the issue of the allocation of deceased donor organs. In the only substantial study, 128 transplant recipients and 104 haemodialysis patients selected which of two hypothetical patients should receive a deceased donor kidney in each of eight scenarios designed to assess an aspect of the UK allocation scheme [17]. Despite patient representation in the decision making process of such policies, there was evidence of substantial disagreement with such aspects as pre-emptive transplantation and the importance given to HLA matching, recipient age, and balance of exchange arrangements. Therefore, the interpretation of justice and equity, and application to transplantation, may differ substantially between the perspectives of health care professionals and ESRD patients. Some argue that the complexity of these issues, that already chal- lenge health care professionals, cannot be appropriately assessed by patients without substantial additional education and that the conclusions drawn are therefore of questionable validity. While patients must be partners in the development of transplant allocation policies, their representatives should have adequate information to allow an appropriate and meaningful contribution and avoid the addition of bias to an already challenging area of debate.

\section{Possible Future Options}

Some advocate transplanting those kidneys with a projected shorter lifespan into recipients with a predicted reduced survival. In the USA, if a policy of 'old for old' was adopted, approximately $16 \%$ of donated kidneys would be reallocated with the mean gain of survival per transplant in this cohort estimated at 3 years [18]. While giving 'lower-quality' organs to the older age group raises further moral and ethical arguments, it should be noted that it is already internationally acceptable to give preference in allocation policies to an age-defined subpopulation, children and adolescents, to the detriment of other age groups. It could be argued that the current system is inequitable to younger patients who have less opportunity for dialysis-free survival (by receiving organs from older donors) than older individuals who receive organs with greater projected survival than the recipient. The policy of older donors for older recipients may already exist in practice by the decision-making of local transplant centres in the acceptance and allocation of organs; the frequency of patients older than 55 years receiving organs from donors in the same age category was $46 \%$ higher than what would be expected by chance if donor and recipient age were random variables [19].

The concept of grading the quality of donor kidneys has been further advanced by the development of a scoring system based on factors known in the peritransplant period to predict the likely graft years available from any particular organ [20]. A recipient score has been proposed with the suggestion that pairing donor organs with recipients based on the projected survivals of both would increase the utility of deceased donor grafts [9]. There has not been widespread adoption of this system, but in the USA there is increasing interest in using net survival benefit, the expected gain of years by receiving a transplant compared to remaining on dialysis, in allocation decisions [16]. Yet any formulaic determination of estimated life expectancy, much less life quality, based on population studies is difficult to apply to an individual patient, 
and any change to allocation schema cannot benefit all wait-listed patients. Given the shortage of organs the transplant community has a responsibility to use the bequeathed organs to their maximum potential, considering such systems as the 'gain in years principle' or donor and recipient scoring, while maintaining as much as possible the equipoise between utility and equity.

\section{Conclusion}

There is a substantial difference between the relative and absolute benefits for transplantation in different groups, such as persons who are older, with DM, who are obese, or highly sensitised. For each individual in these cohorts there is a survival benefit in having a successful transplant. For the ESRD population, and society at large, however, the maximal potential benefit from the altruistic act of the donation would be reached only if it were transplanted into a young recipient without co-morbidity.
Modification of allocation policies to enhance utility may reduce equity of access. This does not make such policies unethical. Maintaining the principle of equity to the minimisation of utility is in itself unjustifiable in this era of increasing disparity between the numbers waiting for a kidney transplant and the static nature of the deceased donor organ pool. There is a persistent challenge to maintain a socially acceptable equipoise between utility and equity. With promotion of organ donation, from both living and deceased donors, all involved in the transplant process must strive to 'do what is right' within their own sphere of practice. While subject to the national allocation system, we advocate on-going regional review of the transplant listing strategy and the policy regarding acceptable grafts. Involvement of local patient representatives will avoid paternalism and include an important potentially alternative interpretation of these ethical principles.

\section{References}

1 Wolfe RA, Ashby VB, Milford EL, Ojo AO, Ettenger RE, Agodoa LY, Held PJ, Port FK: Comparison of mortality in all patients on dialysis, patients on dialysis awaiting transplantation, and recipients of a first cadaveric transplant. N Engl J Med 1999;341:1725-1730.

2 Norman DJ: The kidney transplant wait-list: allocation of patients to a limited supply of organs. Semin Dial 2005;18:456-459.

3 Gutmann T, Daar AS, Sells RA, Land W: Ethical, Legal and Social Issues in Organ Transplantation. Lengerich, Pabst Science Publishers, 2004.

-4 McGeown MG, Kennedy JA, Loughridge WG, Douglas J, Alexander JA, Clarke SD, McEvoy J, Hewitt JC: One hundred kidney transplants in the Belfast city hospital. Lancet 1977;ii:648-651.

5 Danovitch G, Savransky E: Challenges in the counseling and management of older kidney transplant candidates. Am J Kidney Dis 2006;47(suppl 2):S86-S97.

6 Gore JL, Pham PT, Danovitch GM, Wilkinson AH, Rosenthal JT, Lipshutz GS, Singer JS: Obesity and outcome following renal transplantation. Am J Transplant 2006;6: 357-363.

7 Meier-Kriesche HU, Arndorfer JA, Kaplan B: The impact of body mass index on renal transplant outcomes: a significant independent risk factor for graft failure and patient death. Transplantation 2002;73:70-74.
8 Glanton CW, Kao TC, Cruess D, Agodoa LY, Abbott KC: Impact of renal transplantation on survival in end-stage renal disease patients with elevated body mass index. Kidney Int 2003;63:647-653.

-9 Baskin-Bey ES, Kremers W, Nyberg SL: A recipient risk score for deceased donor renal allocation. Am J Kidney Dis 2007;49:284293.

10 Rudge C, Johnson RJ, Fuggle SV, Forsythe JL: Renal transplantation in the United Kingdom for patients from ethnic minorities. Transplantation 2007;83:1169-1173.

11 Schwartz J, Stegall MD, Kremers WK, Gloor $\mathrm{J}$ : Complications, resource utilization, and cost of ABO-incompatible living donor kidney transplantation. Transplantation 2006; 82:155-163.

12 Rudge CJ, Fuggle SV, Burbidge KM: Geographic disparities in access to organ transplantation in the United Kingdom. Transplantation 2003;76:1395-1398.

13 Organ trafficking and transplant tourism and commercialism: the Declaration of Istanbul. Lancet 2008;372:5-6.
14 Schold JD, Kaplan B, Chumbler NR, Howard RJ, Srinivas TR, Ma L, Meier-Kriesche HU: Access to quality: evaluation of the allocation of deceased donor kidneys for transplantation. J Am Soc Nephrol 2005; 16:31213127.

15 Segev DL, Simpkins CE, Thompson RE, Locke JE, Warren DS, Montgomery RA: Obesity impacts access to kidney transplantation. J Am Soc Nephrol 2008;19:349-355.

16 Stegall MD: The development of kidney allocation policy. Am J Kidney Dis 2005;46: 974-975.

17 Geddes CC, Rodger RS, Smith C, Ganai A: Allocation of deceased donor kidneys for transplantation: opinions of patients with CKD. Am J Kidney Dis 2005;46:949-956.

18 Meier-Kriesche HU, Schold JD, Gaston RS, Wadstrom J, Kaplan B: Kidneys from deceased donors: maximizing the value of a scarce resource. Am J Transplant 2005;5: 1725-1730.

19 Kasiske BL, Snyder J: Matching older kidneys with older patients does not improve allograft survival. J Am Soc Nephrol 2002;13: 1067-1072.

-20 Nyberg SL, Matas AJ, Kremers WK, Thostenson JD, Larson TS, Prieto M, Ishitani MB, Sterioff S, Stegall MD: Improved scoring system to assess adult donors for cadaver renal transplantation. Am J Transplant 2003;3: 715-721. 


\section{Editorial Comment}

M. El Nahas, Sheffield

This timely review discusses issue related to the challenges of equity in provision of organs for kidney transplantation. Globally, the shortage of organs for kidney transplantation has challenged medical and national health care authorities to come up with policies to address this issue without compromising ethics and human rights. The review examines some of the allocation schemes and national policies including approaches based on commoditisation (ability to pay), waiting time, chance/random allocation as well as maximum benefit (greatest good to the greatest number). It also discusses ethical considerations linked to some of these schemes including the Istanbul Declaration of 2008; the latter concluded that organ trafficking and transplant tourism violate the principles of equity, justice and respect for human dignity and should be prohibited.
It is important to bear in mind that geographical and socio-economical factors along with local cultural influences have considerable impact on transplantation practices worldwide. All too often some in a given societal culture condemn, with little understanding, those practicing in a different cultural environment. With that in mind, I recall André Malraux' quote: 'Juger c'est mal comprendre' (To judge is to misunderstand), from $L a$ Condition Humaine.

Guidelines in the field of equity and access to transplantation have to be guided by good clinical practice and an utmost and profound respect of human rights; those of the donors and the recipients. 